\title{
Electromagnetic receiver with capacitive electrodes and triaxial induction coil for tunnel exploration
}

\author{
Chen Kai ${ }^{1}$, Jin Sheng ${ }^{1 *}$ and Shun Wang ${ }^{2}$
}

\begin{abstract}
A new type of electromagnetic (EM) receiver has been developed by integrating four capacitive electrodes and a triaxial induction coil with an advanced data logger for tunnel exploration. The new EM receiver can conduct EM observations in tunnels, which is one of the principal goals of surface-tunnel-borehole EM detection for deep ore deposit mapping. The use of capacitive electrodes enables us to record the electrical field (E-field) signals from hard rock surfaces, which are high-resistance terrains. A compact triaxial induction coil integrates three independent induction coils for narrow-tunnel exploration applications. A low-time-drift-error clock source is developed for tunnel applications where GPS signals are unavailable. The three main components of our tunnel EM receiver are: (1) four capacitive electrodes for measuring the E-field signal without digging in hard rock regions; (2) a triaxial induction coil sensor for audio-frequency magnetotelluric and controlled-source audio-frequency magnetotelluric signal measurements; and (3) a data logger that allows us to record five-component MT signals with low noise levels, low time-drift-error for the clock source, and high dynamic range. The proposed tunnel EM receiver was successfully deployed in a mine that exhibited with typical noise characteristics.
\end{abstract}

Keywords: Tunnel EM prospecting, Capacitive electrode, Triaxial induction coil, Low time-drift-error clock source, EM data logger

\section{Introduction}

Electromagnetic (EM) sounding methods were originally developed for imaging metal ores, underground water, active faults, etc. (Kanda and Ogawa 2014). EM geophysical methods require accurate and reliable measurement of the electric field (E-field) and magnetic field (B-field) in order to characterize the subsurface, from the near surface down to a depth of several kilometers. EM sounding methods are ideal for mineral exploration applications and can potentially map the size, depth, strike, and dip of conductors. While surface EM sounding is useful for the exploration of deep electrical anomalies, the effective prospecting depth is limited by weak surface anomalies. If application of a controlled source to the surface and borehole and receive a controlled-source

\footnotetext{
*Correspondence: jinsheng@cugb.edu.cn

${ }^{1}$ China University of Geosciences, Beijing, China

Full list of author information is available at the end of the article
}

EM signal from the surface, tunnel, and borehole, the effective anomalies may be high. Surface-tunnel-borehole electromagnetic (STBEM) surveys in reconnaissance drill holes increase the effective penetration depth; provide the direction and distance to a nearby missed conductor, including the distances to the edges and centers of deposits around boreholes; and improve surface EM interpretation in a prospect area. Downhole EM surveys of deep drill holes can yield precise information about the position and conductivity of nickel sulfide. Crone Geophysics offers a full suite of EM sensors for both borehole and surface surveys, which are very useful in mine development and production applications, specifically for defining the boundaries and sizes of conductive ore zones (Crone Geophysics 2017). The SMARTem Electrical Methods Geophysical Receiver System has evolved as a flexible new tool for time-domain EM (TEM) sounding, induced polarization (IP) sounding, and other electrical geophysical survey methods (Stolz 2000). SMARTem has 
been used in fixed-loop, moving-loop, borehole (axial and three-component), and underground surveys, in both direct-trigger and crystal-synchronized modes.

For deep metal mineral exploration, we developed an STBEM system. Figure 1 presents the experimental layout of our STBEM survey, which uses many surface EM transmitters in different directions as well as borehole EM transmitters. Many surface EM receivers, tunnel EM receivers, and borehole EM receivers are deployed in an array. All recording units are permanently synchronized to the global positioning system (GPS) time. They are optimized to operate with transmitters similarly synchronized to GPS time and are clocked using inbuilt clock source modules. Depending on the instrument, we can conduct surveys in the tunnel using conventional methods such as audio-frequency magnetotellurics (AMT), controlled-source AMT (CSAMT), or spectral IP (SIP).

Existing surface EM receivers, such as ADU from Metronix (Metronix 2017), GDP 32 from Zonge (Zonge 2017), and V8 from Phoenix Geophysics (Phoenix Geophysics 2017b), demonstrate multiple functions, multiple channels, broad bandwidths, and low clock errors using induction coils or fluxgate sensors and non-polarization electrodes. However, when applied to EM signal recording in tunnels, the conventional surface EM receivers have a few limitations. (1) Because of the highly resistive terrains in the tunnel hard rock surface, non-polarization electrodes have significant measurement and operational limitations. (2) The existing induction coils are too long to fit in the narrow tunnel space. (3) An effective GPS signal may not be available in the tunnel. Therefore, the conventional time-synchronized solution may not meet the ultra-low clock drift error requirements; the

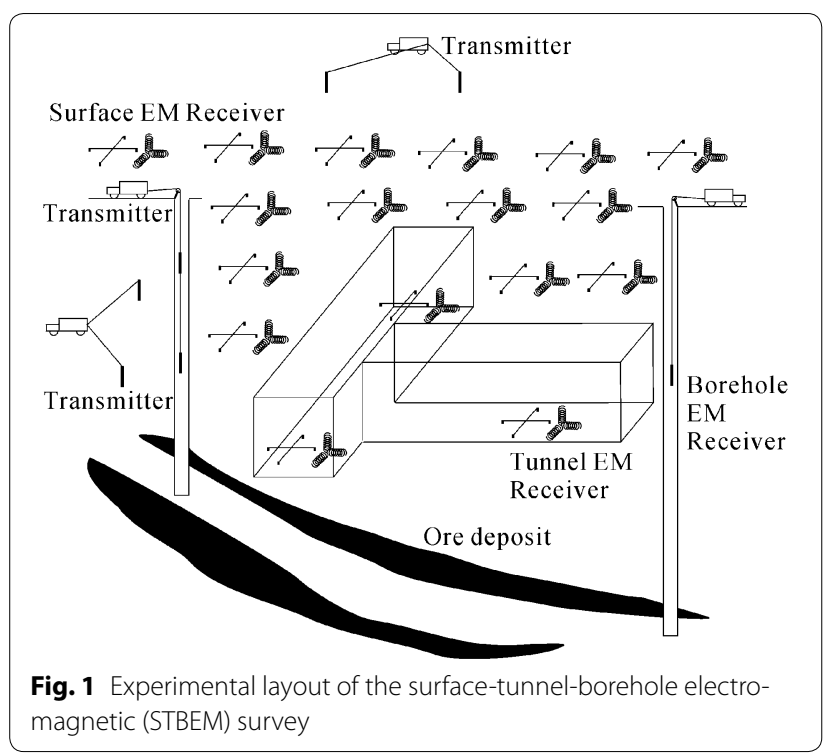

time-synchronized error depends on the clock drift of the clock module.

As a suitable EM receiver for tunnel EM survey targets had not yet been developed, we decided to develop a new tunnel EM receiver system with capacitive electrodes, a triaxial induction coil, and a data logger with a low timedrift-error clock source. The key features of the tunnel EM receiver system we developed are (1) capacitive electrodes for highly resistive terrains in the audio-frequency band, (2) a compact triaxial audio-frequency induction coil with low noise levels, and (3) ultra-low clock drift error. Here we present the details of the sensor used in our system, show an outline of the new instruments, and present the preliminary field data.

\section{Instruments}

The tunnel EM receiver mainly consists of four capacitive electrodes, a triaxial induction coil, and a data logger. These three parts will be itemized in the following subsections, together with their general features. Table 1 summarizes the specifications of the tunnel EM receiver.

\section{General design}

Figure 2 shows the block diagram of the tunnel EM receiver layout. Four capacitive electrodes are laid out orthogonally for the horizontal E-field component measurement. The electrode dipole length depends on the tunnel space. A triaxial induction coil integrates three independent audio-frequency MT induction coils in a $30 \mathrm{~cm} \times 30 \mathrm{~cm} \times 30 \mathrm{~cm}$ cube. The triaxial induction coil is designed to ensure an overall noise level less than $1 \mathrm{pT} / \sqrt{ } \mathrm{Hz}$ at $1 \mathrm{~Hz}$ in a volume of $30 \mathrm{~cm} \times 30 \mathrm{~cm} \times 30 \mathrm{~cm}$ with a weight less than $8 \mathrm{~kg}$. In order to avoid disturbing the sensitive induction coil, the data logger is placed away from the triaxial induction coil sensor. The coil is located $3 \mathrm{~m}$ away from the data logger. The data logger is integrated with a GPS antenna. Before conducting

\begin{tabular}{ll}
$\begin{array}{l}\text { Table } 1 \text { Specifications } \\
\text { receiver }\end{array}$ & the electromagnetic (EM) \\
\hline Number of channels & $5(\mathrm{Ex} / \mathrm{Ey} / \mathrm{Hx} / \mathrm{Hy} / \mathrm{Hz})$ for AMT and CSAMT \\
& $3(\mathrm{E} 1 / \mathrm{E} 2 / \mathrm{E} 3)$ for SIP \\
E-field: $1 \mu \mathrm{V} / \sqrt{ } \mathrm{Hz}$ at $1 \mathrm{~Hz}$ \\
Noise level & $\mathrm{B}-\mathrm{field}: 1 \mathrm{pT} / \sqrt{ } \mathrm{Hz}$ at $1 \mathrm{~Hz}$ \\
& $\mathrm{DC}-10 \mathrm{kHz}$ \\
Frequency range & $6 \mathrm{~W}, \mathrm{built}-$ in battery lifetime $>24 \mathrm{~h}$ \\
Power consumption & $<10 \mu \mathrm{s} / 24 \mathrm{~h}$ \\
Clock drift error & $64 \mathrm{~GB}(\mathrm{upgradeable})$ \\
Data storage & $24,000 \mathrm{~Hz} / 2400 \mathrm{~Hz} / 150 \mathrm{~Hz}$ \\
Sample rate & Ethernet $100 \mathrm{Mbps}$ \\
User interface & $117 \mathrm{~dB}(f \mathrm{~s}=2400 \mathrm{~Hz})$ \\
Dynamic range &
\end{tabular}




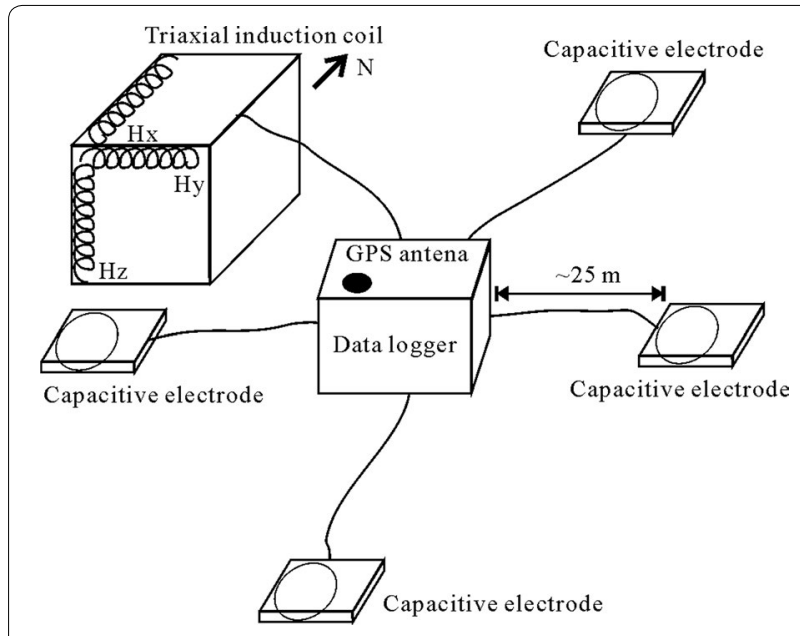

Fig. 2 Block diagram of the electromagnetic (EM) receiver (layout set as audio-frequency magnetotelluric (AMT) and controlled-source audio-frequency magnetotelluric (CSAMT) modules)

the experiment, the data logger locks the GPS onto the surface. While working in a tunnel, the data logger continues recording synchronously using the built-in ultralow-time-drift clock module, and the frequency stability is approximately $5 \times 10^{-11}$. The data logger has a built-in 11.1-V/15-Ah Li-ion battery package instead of the external conventional heavy lead-acid battery.

\section{Capacitive electrode}

The conventional non-polarization electrodes used to measure the E-field have significant achievement (Petiau 2000). However, for tunnel E-field measurements, due to the high-resistance hard rock terrain, the conventional $\mathrm{PbCl}_{2}$ electrodes have operational limitations. Besides, the conventional $\mathrm{PbCl}_{2}$ electrodes may be affected by temperature variations, ionic concentration, or corrosion. As capacitive coupling is a purely EM phenomenon, it can provide stable measurement. The capacitive electrodes allow EM surveys to be conducted in tunnels where such measurements were previously difficult or impossible.

To resolve signals using small coupling capacitances, the use of modern integrated amplifiers to achieve ultrahigh input impedances is a valid approach. Capacitive sensor approaches (Wang et al. 2016, Hibbs et al. 2011, Matthews et al. 2005) have been successfully conducted for geo-electric field recordings. The general measurement circuit architecture for capacitive sensing is shown in Fig. 3. There is no resistive path to the ground at the input to carry the amplifier input bias current. As a result, the current flows into the electrode capacitance $C_{s}$, increasing the voltage at the amplifier input until it saturates. In order to stabilize the amplifier, an amplifier

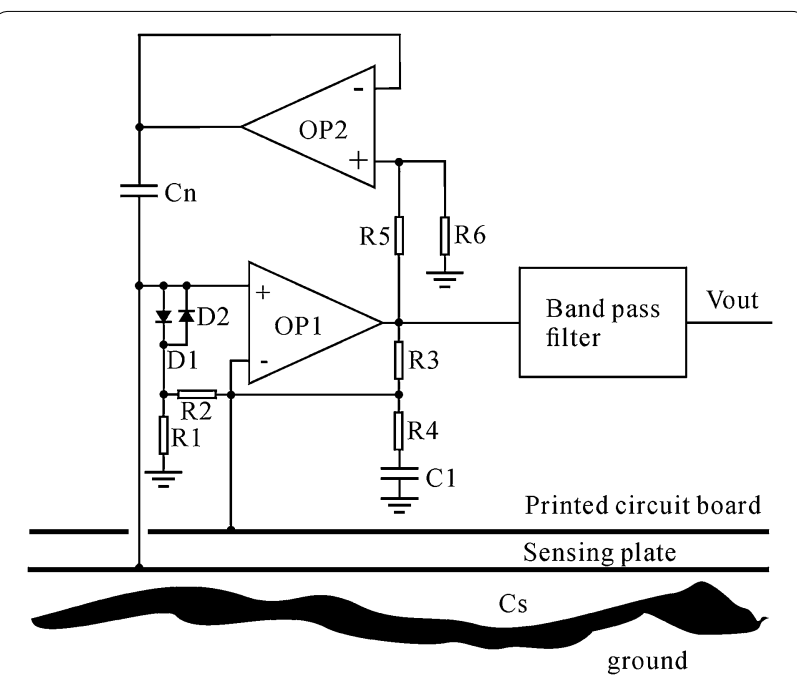

Fig. 3 Diagram of the capacitive electrode. The electrode consists of an analog amplifier and a standard printed circuit board. The sensing plate (diameter $15 \mathrm{~cm}$ ), along with the analog amplifier OP1, forms the high-impedance front end with a capacitance neutralization circuit. The plate capacitor $C_{s}$ is formed by the ground and the plate of the electrode connected to the non-inverting input of OP1. Diodes $D_{1}$ and $D_{2}$ provide a resistive path at the amplifier input without essentially shorting the signal to the amplifier. The gain of the first stage is simply set by $R_{1}$ and $R_{2}$. A well-known active shield is used to avoid current leakage at the input. The voltage divider formed by $C_{s}$ and $C_{\text {in }}$ has a detrimental effect on the gain and channel matching. To address this, $R_{5}$ and $R_{6}$ are adjusted to arrive at values such that the input capacitance is almost completely negated, resulting in an amplifier gain that is essentially invariant with respect to $C_{\mathrm{s}}$. The corner frequency of the band-pass filter is set between $0.1 \mathrm{~Hz}$ and $100 \mathrm{kHz}$

with ultra-high input impedance is required to be useful as a circuit that removes the input bias current from the input potential. In order to avoid saturation during purely capacitive measurements, a feedback loop is designed to provide a bias current path to the ground without reducing the input impedance of the amplifier. The capacitive electrode can provide immunity to changes in ground resistance. A guard creates an equal potential zone around the input pins, preventing external leakages from reaching the input. The guard is driven to the same potential as the inverting input node. Figure 4 shows a photograph of a capacitive electrode and a conventional $\mathrm{PbCl}_{2}$ electrode. The capacitive electrode contains an electrode box and a battery box, which supplies power for the electronic components. The power consumption is approximately $\pm 2 \mathrm{~mA}$ at $3.6 \mathrm{~V}$.

\section{Triaxial induction coil}

It is well known that an induction coil is used for surface MT signal measurements. For tunnel EM measurement applications, the length of the induction coil is limited 


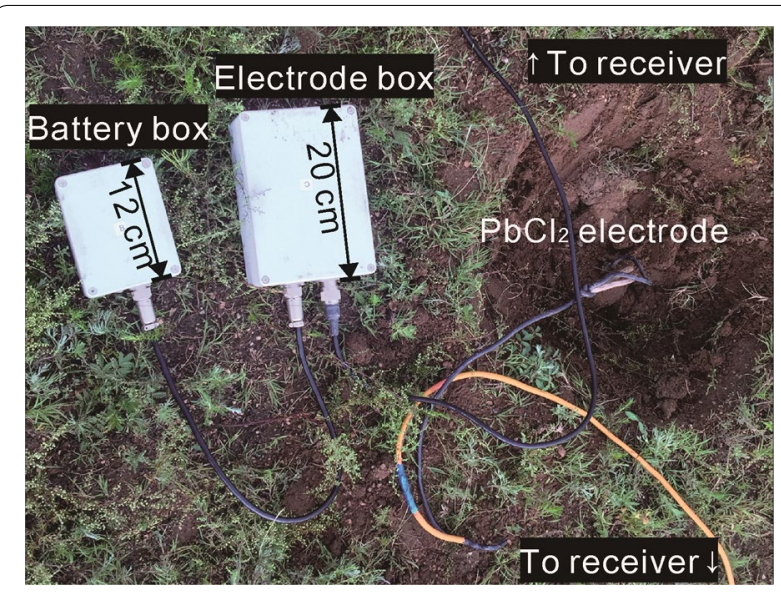

Fig. 4 Photographs of the capacitive electrode and $\mathrm{PbCl}_{2}$ electrode

by the narrow tunnel space. A fluxgate sensor is compact, but the noise level and working frequency range are inadequate. Compared to the fluxgate sensor, the noise level of the induction coil is lower in the audio-frequency range. Currently, commercial and laboratory-developed induction coils for the AMT method are mostly thin with a length of generally $1 \mathrm{~m}$ and a diameter of approximately $5-10 \mathrm{~cm}$. For example, for the Phoenix Geophysics AMTC-30, the noise level is $1 \mathrm{pT} / \sqrt{ } \mathrm{Hz}$ at $1 \mathrm{~Hz}$, length is $0.82 \mathrm{~m}$, and diameter is $6 \mathrm{~cm}$. In the case of limited tunnel space, if we integrate three orthogonal induction coils in a small cube, we can measure the AMT signals within a compact area and with low noise levels. The triaxial induction coil allows us to integrate three orthogonal coils in a single assembly with a very high volume utilization factor. A much more compact design of induction coils was proposed by Roux et al. (2008), wherein the coils were thick but short, contrary to the traditional designs wherein the coils were thin but very long. However, the frequency range was not wide enough. Grosz et al. (2011) designed a triaxial induction coil magnetometer to achieve compact size and low power consumption. However, the noise level $(12 \mathrm{pT} / \sqrt{ } \mathrm{Hz}$ at $1 \mathrm{~Hz})$ was too high for geophysical survey applications.

Hence, we optimized the induction coils based on the requirements of compact size and low noise level. The optimization goal was to reach a noise level and sensitivity comparable to that of the AMTC-30 (Phoenix Geophysics 2017a). We redesigned the induction coil to integrate three independent induction coils in a compact cube based on a study by Bin et al. (2013), which uses the magnetic flux concentrator technology. To reach this aim, we optimized the coil parameters considering this new constraint. The optimization provided the parameters of the low-noise preamplifier and flux concentrators. The triaxial induction coil dimensions were $30 \mathrm{~cm} \times 30 \mathrm{~cm} \times 30 \mathrm{~cm}$. Figure 5 presents the block diagram of the triaxial induction coil sensor. All coils and amplifiers are accommodated within a single electrostatic shield and a single housing. Three orthogonal coils with an aspect ratio of 2 very efficiently fill in the total cubic volume of the magnetometer. An aspect ratio close to 2 (13 cm diameter, $28 \mathrm{~cm}$ total length) of the induction coils provides a very high $(70 \%)$ volume utilization factor. The length and diameter of the core are 20 and $1 \mathrm{~cm}$, respectively. The diameter and thickness of the flux concentrators are 11 and $1 \mathrm{~cm}$, respectively. An MnZn ferrite with a relative magnetic permeability of 2000 is selected as the material for the flux concentrators and coil cores. The core and flux concentrators fit tightly around each other. Multi-turn coils are wound around the core. Bipolar junction transistors are used as low-noise preamplifiers. The induction coil is optimized for frequencies ranging from $1 \mathrm{~Hz}$ to $10 \mathrm{kHz}$ and for ultra-low noise levels. All coils are orthogonal to each other. For three orthogonal induction coils, the orthogonal error is less than $\pm 1^{\circ}$.

Figure 6 shows a photograph of the triaxial induction coil. To simplify field operation, a leveling bubble is installed on the top panel. Each induction coil is integrated with a calibrated coil. Thus, a compact and sensitive triaxial induction coil is designed, built, and tested. Figure 7 presents the calibration results. The sensitivity has been calculated as $100 \mathrm{mV} / \mathrm{nT}$ over a flat frequency band. The homogeneity among the induction coils is high. Figure 8 presents the self-noise power spectrum density. The coil noise level is $1 \mathrm{pT} / \sqrt{ } \mathrm{Hz}$ at $1 \mathrm{~Hz}$, similar to that of the Phoenix Geophysics AMTC-30.

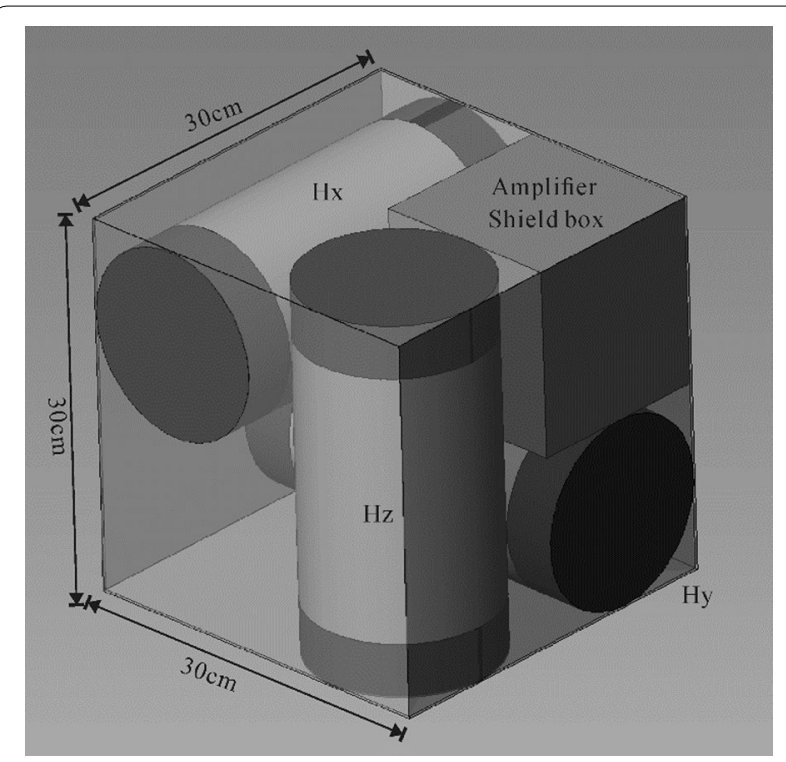

Fig. 5 Block diagram of the triaxial induction coil sensor 

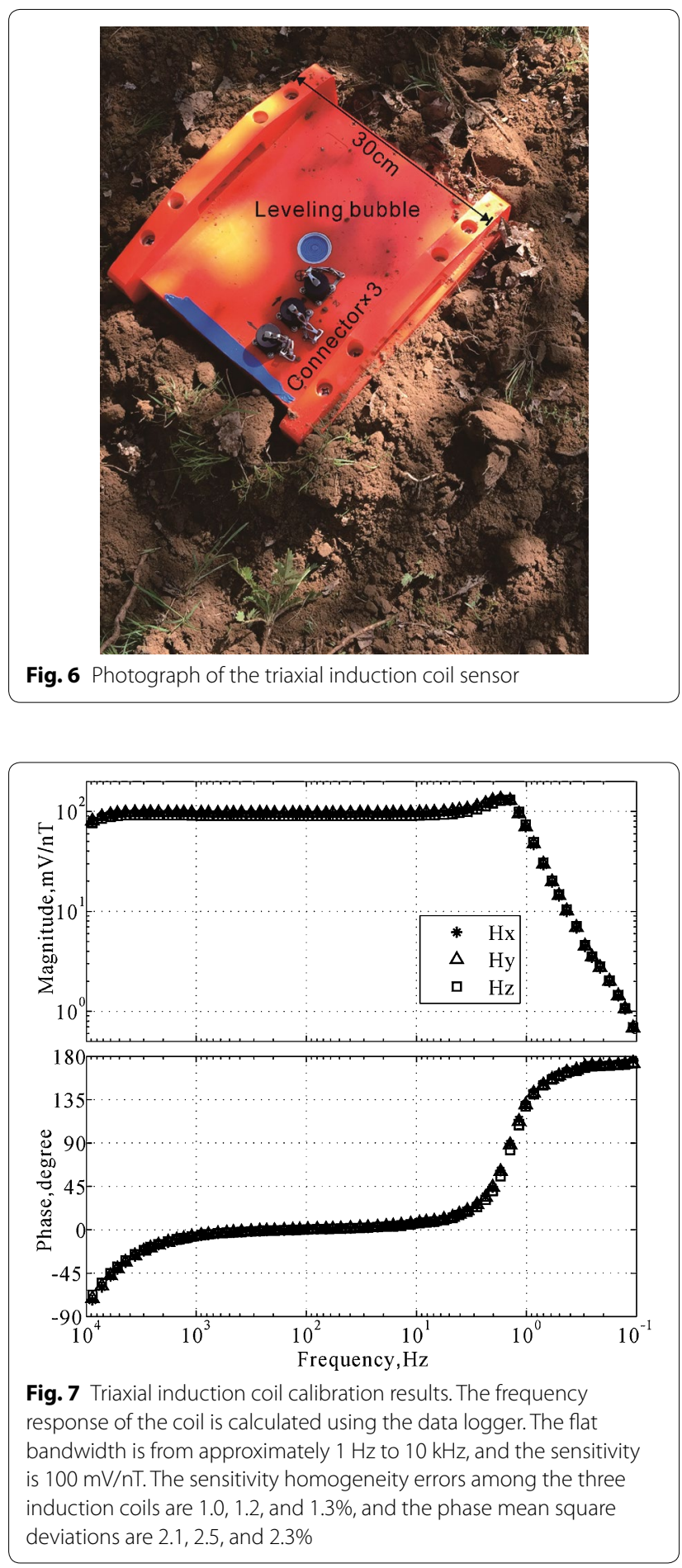

\section{Data logger}

The data logger consists of a built-in recorder circuit, battery package, GPS antenna, and aluminum case. Figure 9 presents a block diagram of the data logger. The recorder circuit integrates six pre-amplifiers, an analogto-digital converter (ADC), a field-programmable gate array (FPGA), a microcontroller unit (MCU), an atomic

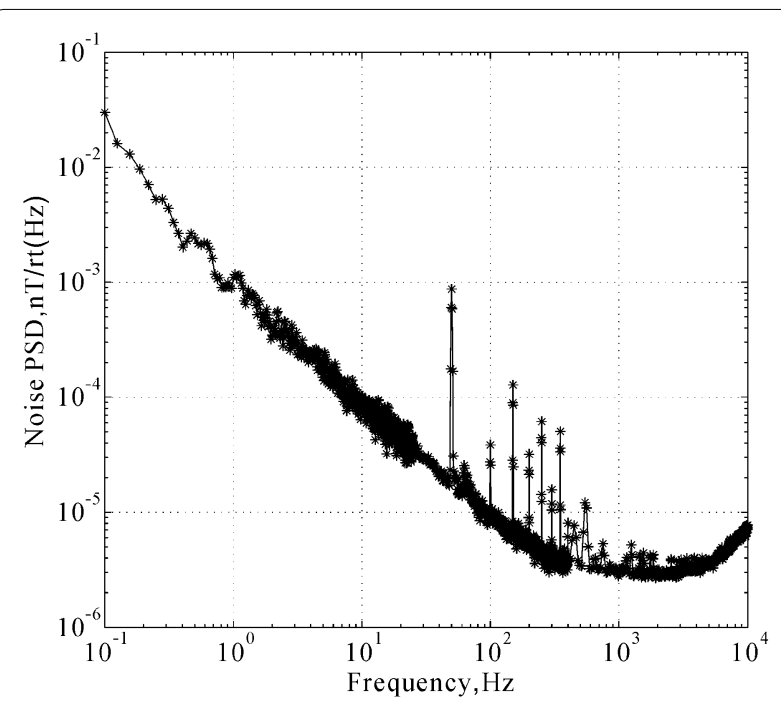

Fig. 8 Self-noise level of the induction coil. The induction coil sensor noise level is measured in a magnetically shield room using a dynamic signal analyzer Agilent 35670A. The noise level is approximately $1 \mathrm{pT} / \sqrt{ } \mathrm{Hz}$ at $1 \mathrm{~Hz}$. There is $1 / \mathrm{f}$ noise in the 1000 to $0.1-\mathrm{Hz}$ frequency band, and in the nearby band of 1000-Hz frequency, the noise level is flat. Peaks at $50 \mathrm{~Hz}$ and its harmonics are clearly observed because of the ambient $50-\mathrm{Hz}$ noise. This is because the magnetically shield room was not very "clear"

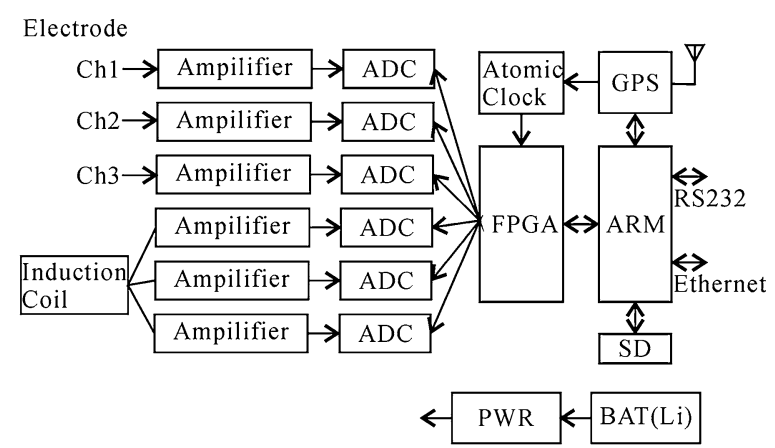

Fig. 9 Block diagram of the data logger

clock module, a GPS module, a power circuit, and an SD card. The amplifier is designed as a low-noise highprecision amplifier. The data logger contains six channels, consisting of three E-field channels and three B-field channels. The five channels comprise two E-field channels and three B-field channels, designed for the AMT and CSAMT modules, and three E-field channels designed for the three E-dipole signals in the SIP module. Each channel assembles a 24-bit ADC. The FPGA is used to package the data stream; it acts as a digital filter and performs time synchronization. The MCU is used for data acquisition control and data storage to the SD card. The SD card space is $64 \mathrm{~GB}$ and is 
upgradeable. The RS232 interface is used for system debugging, and the Ethernet is used for the user interface. The data transfer rate reaches $100 \mathrm{Mbps}$ without any acquisition pause or shutdown record circuit. There is an $11.1-\mathrm{V} / 15-\mathrm{Ah}$ Li-ion battery package inside the aluminum case. The battery lifetime is more than $24 \mathrm{~h}$ if the power consumption is less than $6 \mathrm{~W}$. When working in a tunnel, an external heavy lead-acid battery is unnecessary.

The maximum sample rate of the data logger is $24 \mathrm{kHz}$, and the bandwidth is $\mathrm{DC}-10 \mathrm{kHz}$. The data throughput rate reaches $388 \mathrm{kB} / \mathrm{s}$ (approximately $500 \mathrm{MB} / \mathrm{h}$ ) for the AMT module. The data logger uses direct memory access (DMA) and a double buffer for achieving high data throughout rate. The built-in digital filter is designed to suppress $50 \mathrm{~Hz}$ and its harmonics. An attenuation of $-60 \mathrm{~dB}$ is achieved at $50 \mathrm{~Hz}$ using the digital filter.

For the SIP method module measurement, the maximum transmitter frequency is $128 \mathrm{~Hz}$. The receiver and the electrical current data logger beside the transmitter must be time-synchronized, as the clock drift error will cause a phase error. For example, for a 10-millirad phase error at $128 \mathrm{~Hz}$, the time synchronization error must be less than $12.4 \mu \mathrm{s}$. If the receiver is to operate in the tunnel for $10 \mathrm{~h}$ continuously, the frequency stability must be less than $3.4 \times 10^{-10}$. Conventional oven-controlled crystal oscillators (OCXOs) may not meet this high requirement. The data logger maintains the time synchronization using a GPS and an atomic clock module (SA.45 s CSAC) from Symmetricom. The GPS module (LEA-6T from U-blox) provides excellent navigation performance. Accuracy of up to $20 \mathrm{~ns}$ is achievable using the quantization error information to compensate for the granularity of the pulse per second (PPS). The frequency stability of the atomic clock can be $1 / 100$ th of that of a good OCXO, which means that the time-stamping errors caused by drift are reduced greatly. The SA.45 s provides $10-\mathrm{MHz}$ and 1-PPS outputs at standard CMOS levels, with a short-term stability (Allan deviation) of $2 \times 10^{-10}$ at $1 \mathrm{~s}$, long-term aging of $3 \times 10^{-10} /$ month, and a frequency change of $5 \times 10^{-10}$ over an operating temperature range from -10 to $+70{ }^{\circ} \mathrm{C}$. While working in the tunnel, there is no effective GPS signal and the SA.45 s CSAC provides a stable holdover signal clock module to maintain the high-precision clock source and low time-drift-error PPS for time synchronization.

\section{Field experiment}

The tunnel EM receiver thus developed was tested many times in the field. A sensor comparison test was executed at the surface to confirm the functionality of the capacitive electrodes and the triaxial induction coil.

First, the capacitive electrode comparison test is described. The tunnel EM receiver was laid out on the surface for the AMT method measurement. Each E-channel was connected to the $\mathrm{PbCl}_{2}$ electrode and the capacitive electrode. The $\mathrm{PbCl}_{2}$ electrodes were buried approximately $20 \mathrm{~cm}$ below the ground and were wetted with a sufficient amount of brine. The electrode dipole length was set as $50 \mathrm{~m}$, and the dipoles were arranged in a parallel layout. Two parallel induction coils were connected to the data logger B-channels, which were orthogonal to the electrode dipole. All channel gains were set to 4 , and continuous recording was performed for 30 min. Figure 10 shows a time series comparison of the two types of electrodes. In the high- and mid-frequency bands, the homogeneity is high. At low frequencies, the signal-to-noise ratio (SNR) decreases. We estimated the MT responses (apparent resistivity and impedance phase) using the SSMT2000 from Phoenix Geophysics. To calculate the MT responses of the two E-field channels, the $\mathrm{Hx}$ channel data were shared to the $\mathrm{xy}$ and $\mathrm{yx}$ modes. Figure 11 presents the signal power spectrum densities of the two types of electrodes. Figure 12 presents the MT response curves. The sounding curves from the $\mathrm{PbCl}_{2}$ electrode are smooth over the entire frequency range, and those from the capacitive electrode are relatively smooth in the frequency range above $7 \mathrm{~Hz}$. The capacitive electrodes are considered to be capable of

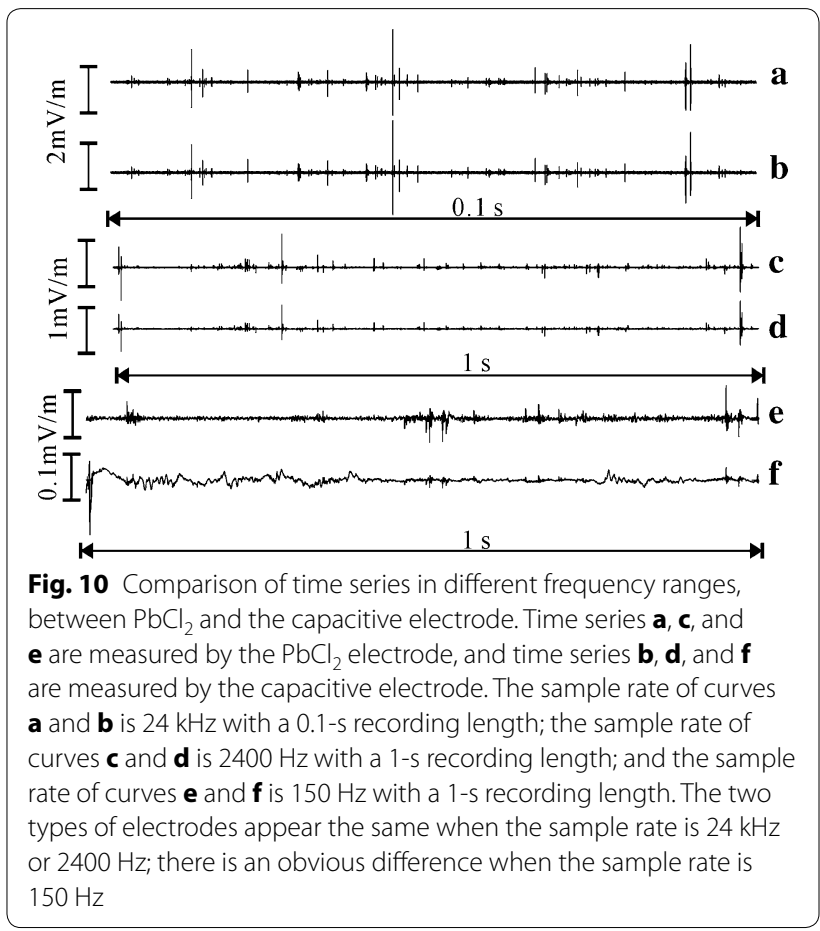




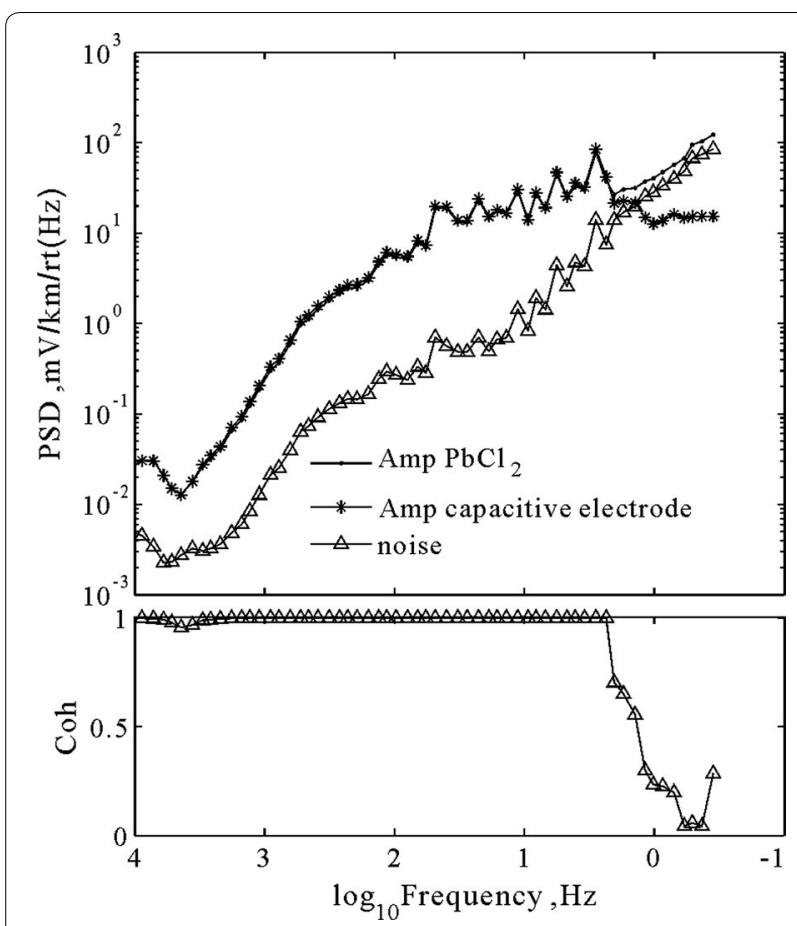

Fig. 11 Results of the noise parallel (NPI) test. From $10 \mathrm{kHz}$ to $3 \mathrm{~Hz}$, the E-field signals measured by the two types of electrodes show high homogeneity. The coherence is high above $3 \mathrm{~Hz}$. Below $3 \mathrm{~Hz}$, the difference in the power spectrum density (PSD) is larger and the coherence decreases. Because the signal to noise ratio (SNR) in the low-frequency bands is low, the data quality decreases. The coherence of the two types of electrodes is approximately 1 , with a fluctuation less than $5 \%$ when the frequency ranges from $3 \mathrm{~Hz}$ to $10 \mathrm{kHz}$

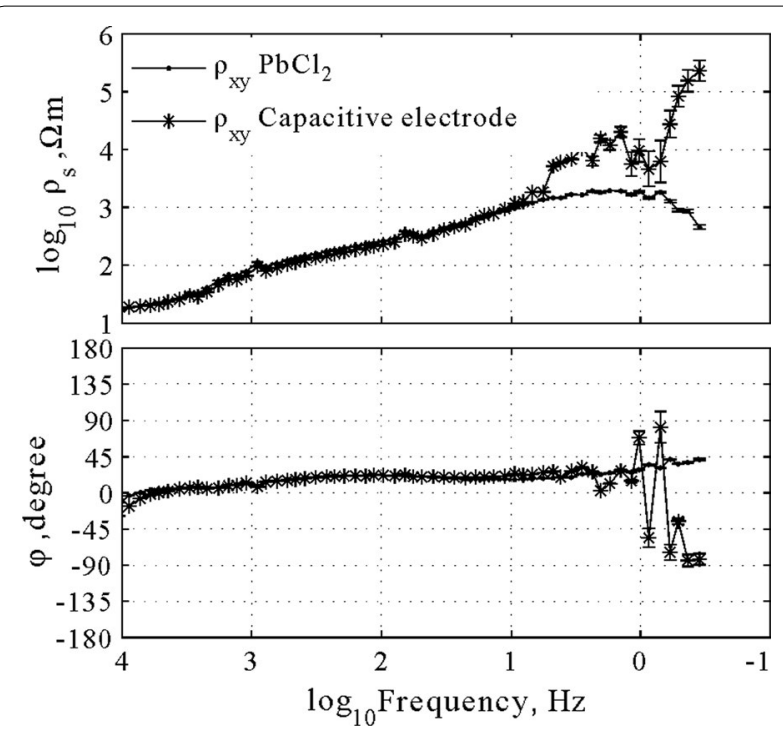

Fig. 12 MT response sounding curves of the two types of electrodes. Apparent resistivity and impedance phases are calculated using the SSMT2000 data-processing software. The two E-field channels are laid out in parallel and share the $\mathrm{Hx}$ channel accurately measuring the natural geo-electric field in the frequency range from $7 \mathrm{~Hz}$ to $10 \mathrm{kHz}$. At lower frequencies, the amplitude bias increases. The intrinsic noises of the capacitive electrodes are almost inversely proportional to the frequency at lower frequencies.

Second, to confirm the functionality of the triaxial induction coil, we deployed two sets of tunnel EM receivers in a field test. One was connected to three AMTC-30 coils (Phoenix Geophysics), and the other was connected to the developed triaxial induction coil. All E-field channels were connected to the conventional $\mathrm{PbCl}_{2}$ electrodes. The AMT mode was recorded continuously for $30 \mathrm{~min}$. Figure 13 presents the MT response calculation results. In the frequency range from $1 \mathrm{~Hz}$ to $10 \mathrm{kHz}$, the homogeneity is high. The performances of the triaxial induction coil and AMTC-30 are similar over the entire frequency range. The data from the triaxial induction coil are assumed to be accurate.

Third, we conducted measurements using the tunnel EM receiver with capacitive electrodes and triaxial induction coil for the CSAMT method in a mine. The mine was located in Linxi County of Inner Mongolia and contained $\mathrm{Pb} / \mathrm{Zn} / \mathrm{Ag} / \mathrm{Cu} / \mathrm{Sn}$ multi-metal mineral deposits. The explored depth was approximately $400 \mathrm{~m}$, and a lot of clutter and piping were present in this region. While the orthogonal components of the EM field were measured using the high-frequency EM method, the tensor measurements of the electrical and magnetic field components were used in the CSAMT method. Before CSAMT data acquisition, we evaluated the environment EM noise using the AMT measurement method. The data were reformatted from the binary format to Phoenix Geophysics MTU time series format according to the SSMT2000 processing software, a commercial MT software package from Phoenix Geophysics for MT response function computation. Figure 14 presents the Cagniard apparent resistivity. The offset between the south current source dipole line and the survey line was $6 \mathrm{~km}$, and the offset between the east current source dipole line and the survey line was $7 \mathrm{~km}$. The current source dipole lengths were $1200 \mathrm{~m}$. The experimental area was approximately $1.5 \mathrm{~km}^{2}$, and we deployed 90 receiving sites. The transmitter frequency range was from 0.9375 to $9600 \mathrm{~Hz}$, which contained 41 frequency points, and the circulation duration time of the transmitter was $50 \mathrm{~min}$. The transmitter current was approximately 20 A over the flat frequency band.

The data are processed using the CSAMT processing routine based in MATLAB. Digital counts from the raw time series recorded by the receiver are converted to volts using the least count (V/count) of the ADC, and then the data are normalized based on the receiver's dipole length and the amplifier gain to obtain the electric field in $\mathrm{V} / \mathrm{m}$ 

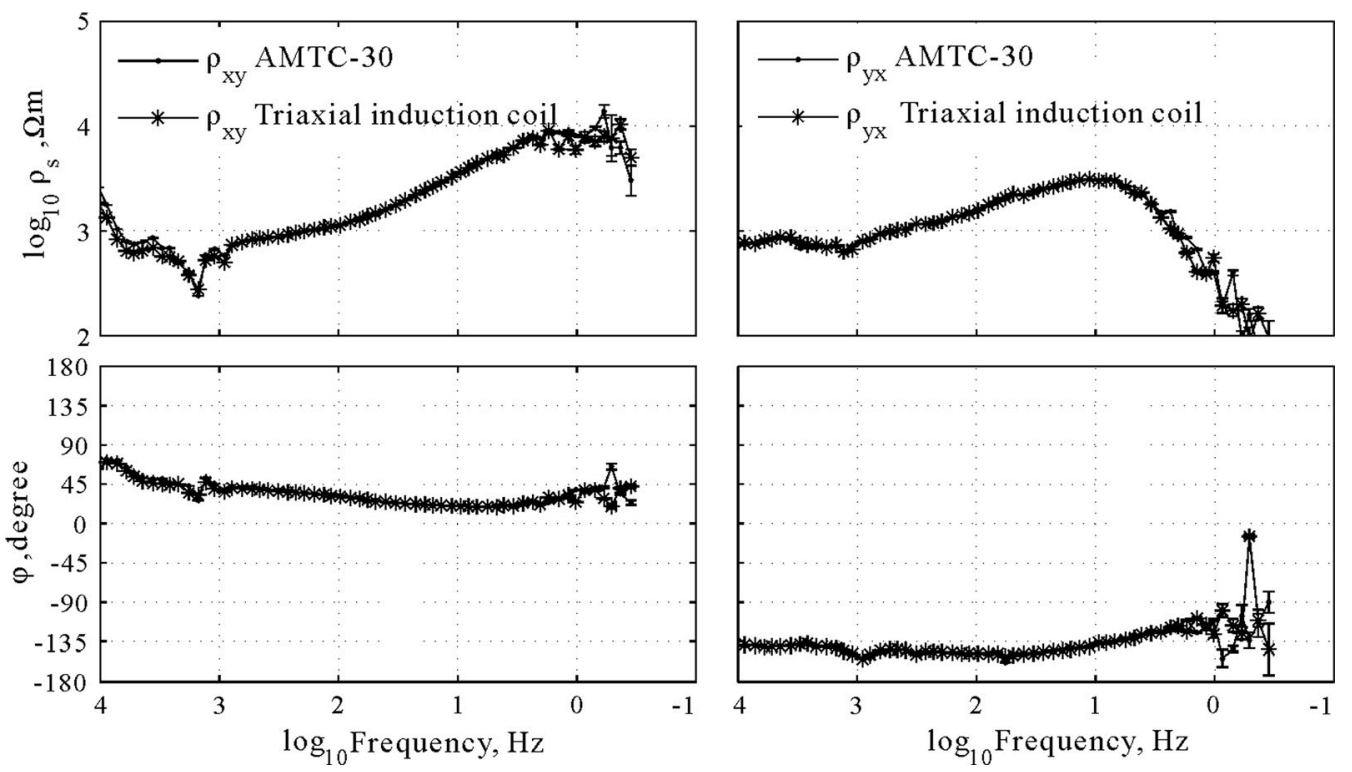

Fig. 13 MT response sounding curves of the two types of induction coils

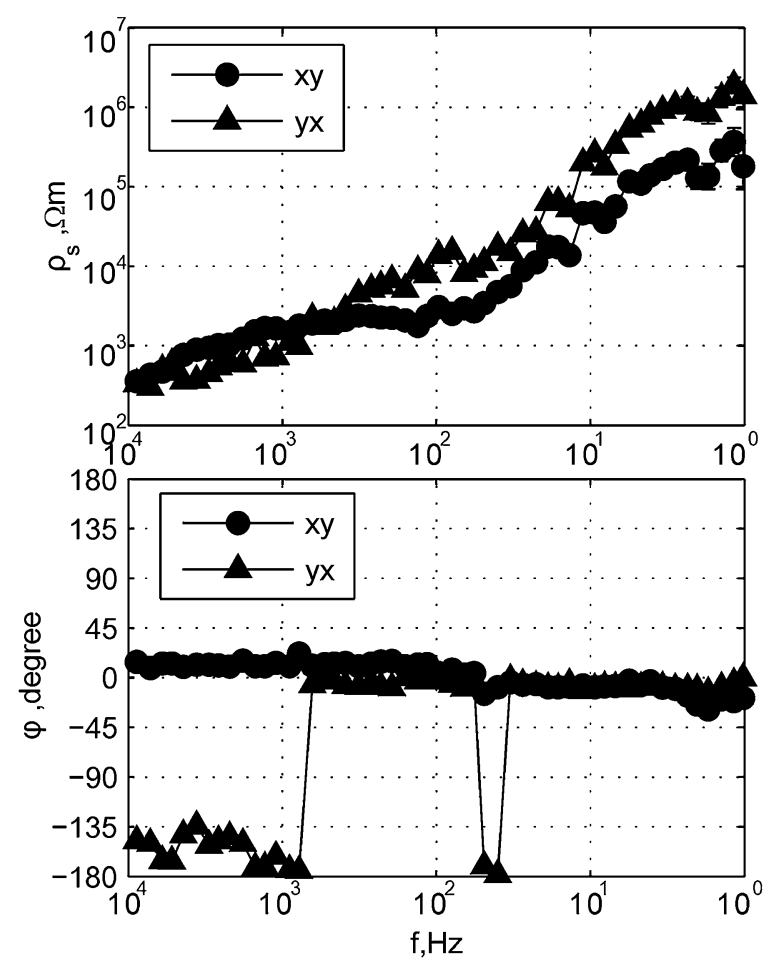

Fig. 14 Mine area EM noise evaluated by the AMT method. The Cagniard apparent resistivity and phase results prove that the nearfield source noise, which is generated by the mine machinery and pipeline, is strong and magnetic field in nT. The data are divided into five stack frames for each frequency. After FFT of the electric and magnetic fields for all stack frames, the power spectrum is computed, and then the impedance tensor is calculated using a robust estimation method. Figure 15 presents the calculated apparent resistivities and the impedance phases of eight sites. The result shows that the stable frequency range is from $0.9375 \mathrm{~Hz}$ to $10 \mathrm{kHz}$. Because of the high SNR of the controlled source signal, the data quality is better than that measured by the AMT method, which records natural sources. Although the data quality in the high-frequency band is improved, strong human activity noises between 100 and $0.9375 \mathrm{~Hz}$ still exist.

\section{Summary}

Compared to the conventional electrodes, this new capacitive electrode could measure the geo-electric field precisely up to $10 \mathrm{kHz}$. In contrast with the $\mathrm{PbCl}_{2}$ electrode, the capacitive electrode could measure the geoelectric field in tunnel applications.

The tunnel EM receiver with capacitive electrodes and a triaxial induction coil was successfully developed and tested experimentally. It enabled E-field measurements in highly resistive tunnel terrains and B-field measurements within limited spaces, with low noise and over broad frequency ranges. AMT and CSAMT experiments indicated 


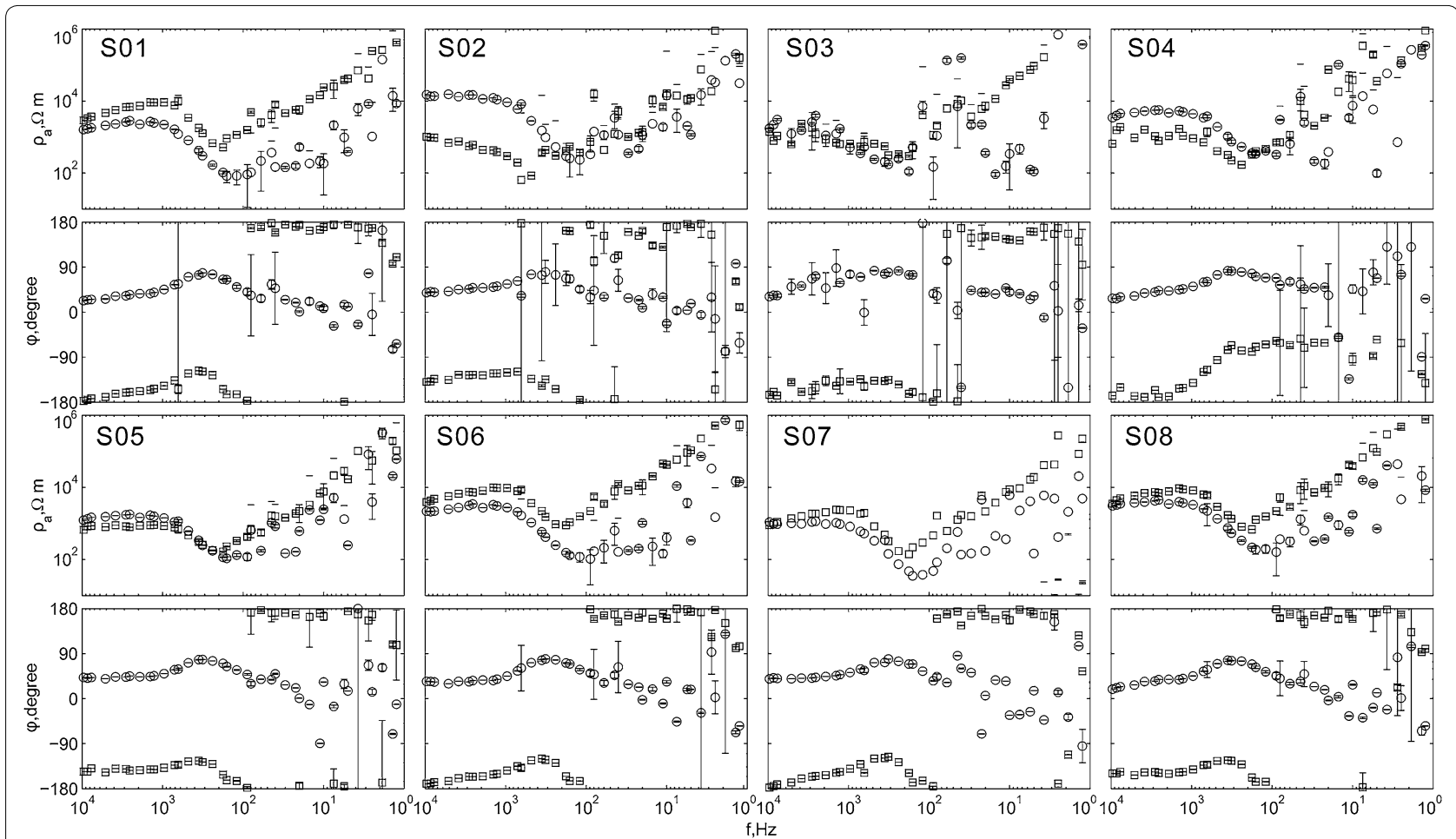

Fig. 15 Tensor CSAMT method module sounding curves in the mine area with strong human activity noises

that the tunnel receiver might be suitable for tunnel EM surveys without requiring corrections for measurement bias.

In the future, the intrinsic noise level of the sensor in the low-frequency band should be decreased. This will not only improve the effective prospecting depth, but it may also be useful for the SIP method module or other low-frequency EM method surveys.

\section{Abbreviations}

EM: Electromagnetic; MT: Magnetotellurics; STBEM: Surface-tunnel-borehole electromagnetic; TEM: Time-domain electromagnetic; IP: Induced polarization; GPS: Global positioning system; AMT: Audio-frequency magnetotellurics; CSAMT: Controlled-source audio-frequency magnetotellurics; SIP: Spectral IP; ADC: Analog-to-digital converter; FPGA: Field-programmable gate array; MCU: Microcontroller unit; OCXO: Oven-controlled crystal oscillator; SNR: Signal-tonoise ratio; FFT: Fast Fourier transformation.

\section{Authors' contributions}

CK developed the required hardware and software. JS created the overall design and performed the field tests. SW was the consultant for capacitive electrode technology. All authors read andapproved the final manuscript.

\section{Authors' information}

KC. was born in JiangXi, China, in 1984. He received his BSc and Ph.D. degrees in Electrical Engineering from the China University of Geosciences, Beijing, China in 2005 and 2016, respectively. From 2008 to present, he has been a researcher with the China University of Geosciences, where he works on geophysical instrument development. His research interests include the development of surface and borehole EM receivers, ocean bottom EM receivers, and EM sensors. J. S. was born in LiangNing, China in 1970. He received his BSC and Ph.D. degrees in geophysics from the China University of Geosciences, Beijing, China in 1994 and 2006, respectively. From 1994 to present, he has been a researcher with the University of Geosciences, where he works on EM method research. Since 2016, he has served as the Director of the Geophysics Institute of the China University of Geosciences. His research interests include EM data processing, inversion, and interpretation. S. W. was born in HeNan, China in 1983. He received his BSc degree in measurement and control technology from the China University of Geosciences, Beijing, China in 2005 and his Ph.D. in EM field and microwave technology from the Chinese Academy of Sciences in 2014. He is a researcher of geophysical instruments.

\section{Author details}

${ }^{1}$ China University of Geosciences, Beijing, China. ${ }^{2}$ Institute of Electronics, Chinese Academy of Sciences, Beijing, China.

\section{Acknowledgements}

We would like to thank WenBo Wei for the top design of the instrument. This work is partly supported by the scientific program " 863 " sponsored by the MOST of China. We would also like to thank GaoFeng Ye and Jianen Jin for their help developing our new system, drawing some figures, and helping prepare the manuscript. The manuscript was greatly improved by the reviewer and editor; two anonymous reviewers provided helpful comments. The SSMT2000 processing software was provided by Phoenix Geophysics Ltd.

\section{Competing interests}

The authors declare that they have no competing financial interests.

\section{Availability of data and materials}

(1) Calibration and noise level of coil sensor, (2) Results of mine field test.

\section{Funding}

The general study and field tests were funded by the National High Technology Research and Development Program of China (2014AA06A603,

2016YFC0303100, 2012AA09A201). The development of the triaxial induction 
coil was supported by the National Science Foundation of China (61531001). The development of the capacitive electrode was supported by the Central University Fundamental Research Project of the Ministry of Education (2652011249, 2652015403)

\section{Publisher's Note}

Springer Nature remains neutral with regard to jurisdictional claims in published maps and institutional affiliations.

Received: 8 February 2017 Accepted: 21 August 2017

Published online: 11 September 2017

\section{References}

Bin Y, Wanhua Z, Leisong L, Kai L, Guangyou F (2013) An optimization method for induction magnetometer of $0.1 \mathrm{mHz}$ to $1 \mathrm{kHz}$. IEEE Trans Magn 49:5294-5300

Crone Geophysics (2017) Pulse-EM methods. vol. 2017, http://www.cronegeophysics.com/pulse-em/methods/. Accessed Jan 01, 2017

Grosz A, Paperno E, Amrusi S, Zadov B (2011) A triaxial search coil magnetometer optimized for small size, low power, and low frequencies. IEEE Sens J 11:1088-1094

Hibbs AD, Dickey RP, Derby K, Petrov T, Lathrop D, Rusakov N, Krupka MA

Markel J (2011) Capacitive electric field measurements for geophysics. In: 73rd EAGE conference and exhibition-workshops 2011
Kanda W, Ogawa Y (2014) Three-dimensional electromagnetic imaging of fluids and melts beneath the NE Japan arc revisited by using geomagnetic transfer function data. Earth Planets Space 66:39

Matthews R, Krupka MA, Hibbs AD (2005) Sensor system for measuring biopotentials. WO2004110268 A1, EP 1631189A1, EP1631189A4, US6961601. US20040254435, 23 Dec 2004

Metronix (2017) Metronix home. http://www.metronix.de/metronix/index php. Accessed Jan 01, 2017

Petiau G (2000) Second generation of lead-lead chloride electrodes for geophysical applications. Pure Appl Geophys 157:357-382

Phoenix Geophysics (2017a) Induction coil sensor. In: Field sensors, vol. 2015, http://www.phoenix-geophysics.com/products/sensors/? Accessed Jan 01,2017

Phoenix Geophysics (2017b) V8 Receiver. vol. 2017, http://www.phoenix-geophysics.com/products/receivers/v8/? Accessed Jan 01, 2017

Roux A, Le Contel O, Coillot C, Bouabdellah A, de la Porte B, Alison D, Ruocco S, Vassal MC (2008) The search coil magnetometer for THEMIS. Space Sci Rev 141:265-275

Stolz EM (2000) Electromagnetic methods applied to exploration for deep nickel sulphides in the Leinster area, Western Australia. Explor Geophys 31:222-228

Wang Z, Wang S, Fang G, Zhang Q (2016) Investigation on a novel capacitive electrode for geophysical surveys. J. Sens 2016:1-9

Zonge (2017) Geophysical data receivers. In: Receivers, http://zonge.com/ instruments-home/instruments/receivers/. Accessed Jan 01, 2017

\section{Submit your manuscript to a SpringerOpen ${ }^{\circ}$ journal and benefit from:}

- Convenient online submission

- Rigorous peer review

- Open access: articles freely available online

- High visibility within the field

- Retaining the copyright to your article

Submit your next manuscript at $\boldsymbol{\nabla}$ springeropen.com 